\title{
Trafficking protein particle complex 6A delta (TRAPPC6AD) is an extracellular plaque-forming protein in the brain
}

\author{
Jean-Yun Chang ${ }^{1}$, Ming-Hui Lee ${ }^{1}$, Sing-Ru Lin ${ }^{1}$, Li-Yi Yang ${ }^{1}$, H. Sunny Sun ${ }^{1}$, \\ Chun-I Sze ${ }^{2}$, Qunying Hong ${ }^{3}$, Yee-Shin Lin ${ }^{4,5}$, Ying-Tsen Chou ${ }^{6}$, Li-Jin Hsu ${ }^{4,5,7}$, \\ Ming-Shiou Jan ${ }^{8}$, Cheng-Xin Gong ${ }^{9}$, Nan-Shan Chang ${ }^{1,5,6,8,10}$ \\ ${ }^{1}$ Institute of Molecular Medicine, National Cheng Kung University Medical College, Tainan, Taiwan, ROC \\ ${ }^{2}$ Departments of Anatomy and Cell Biology, National Cheng Kung University Medical College, Tainan, Taiwan, ROC \\ ${ }^{3}$ Department of Pulmonary Medicine, Zhongshan Hospital, Fudan University, Shanghai, PRC \\ ${ }^{4}$ Department of Microbiology and Immunology, National Cheng Kung University Medical College, Tainan, Taiwan, ROC \\ ${ }^{5}$ Center for Infectious Disease and Signaling Research, National Cheng Kung University Medical College, Tainan, Taiwan, ROC \\ ${ }^{6}$ Institute of Basic Medical Sciences, National Cheng Kung University Medical College, Tainan, Taiwan, ROC \\ ${ }^{7}$ Department of Medical Laboratory Science and Biotechnology, National Cheng Kung University Medical College, Tainan, \\ Taiwan, ROC \\ ${ }^{8}$ Advanced Optoelectronic Technology Center, National Cheng Kung University, Tainan, Taiwan, ROC \\ ${ }^{9}$ Institute of Microbiology and Immunology, Chung Shan Medical University, Taichung, Taiwan, ROC \\ ${ }^{10}$ Department of Neurochemistry, New York State Institute for Basic Research in Developmental Disabilities, Staten Island, \\ NY, USA
}

Correspondence to:

Nan-Shan Chang, e-mail: wox1world@gmail.com

Li-Jin Hsu, e-mail: lijin.hsu@gmail.com

Chun-I Sze, e-mail: chuni.sze@gmail.com

Keywords: Alzheimer's disease, protein aggregation, hippocampus, amyloid beta, TIAF1, TRAPPC6A, WWOX, WOX 1

Received: November 12, $2014 \quad$ Accepted: December 08, $2014 \quad$ Published: February 19, 2015

\section{ABSTRACT}

Tumor suppressor WWOX is involved in the progression of cancer and neurodegeneration. Here, we examined whether protein aggregation occurs in the brain of nondemented, middle-aged humans and whether this is associated with wWOX downregulation. We isolated an $\mathrm{N}$-terminal internal deletion isoform, TPC6A $\Delta$, derived from alternative splicing of the TRAPPC6A (TPC6A) gene transcript. TPC6AD proteins are present as aggregates or plaques in the extracellular matrix of the brain such as in the cortex. Filter retardation assays revealed that aggregate formation of TPC6AD occurs preceding $A \beta$ generation in the hippocampi of middle-aged postmortem normal humans. In a Wwox gene knockout mouse model, we showed the plaques of pT181Tau and TPC6AD in the cortex and hippocampus in 3-week-old mice, suggesting a role of WWOX in limiting TPC6AD aggregation. To support this hypothesis, in vitro analysis revealed that TGF- $\beta 1$ induces dissociation of the ectopic complex of TPC6AD and WWOX in cells, and then TPC6AD undergoes Ser35 phosphorylation-dependent polymerization and induces caspase 3 activation and $A \beta$ production. Similarly, knockdown of WWOX by siRNA resulted in dramatic aggregation of TPC6AD. Together, when WWOX is downregulated, TPC6A $\triangle$ is phosphorylated at Ser35 and becomes aggregated for causing caspase activation that leads to Tau aggregation and $A \beta$ formation.

\section{INTRODUCTION}

Accumulated matrix fibrillar $\beta$-amyloid $(\mathrm{A} \beta)$ plaques and intracellular neurofibrillary tangles (NFT) in the hippocampus has been considered as the hallmark of
Alzheimer's disease (AD) [1-3]. These protein aggregates invoke neuronal death and block neurogenesis and learning and memory capabilities in AD patients. Transforming growth factor beta (TGF- $\beta$ ) has been implicated in the AD pathogenesis [4-6]. TGF- $\beta 1$ causes self-aggregation 
of TIAF1 (12-kDa TGF- $\beta$-induced antiapoptotic factor) in vitro, which leads to degradation of membrane amyloid precursor protein (APP) and generation of $A \beta$ and amyloid fibrils [6]. This in vitro finding positively correlates with the occurrence of in vivo aggregation of TIAF1 in the hippocampi of nondemented, middle-aged humans (age 40-75), and formation of amyloid $\beta$, fibrils and plaques in older AD patients (age 70-95) [6]. TIAF1 aggregates are found in degenerative neurons along the interface between metastatic cancer cells and the brain tissue and in the fibrous tissues of lung cancer [7-9].

Here, we report the isolation of an isoform of TRAPPC6A (TPC6A), known as Trafficking Protein Particle Complex 6A. This isoform TPC6A $\Delta$ possesses an internal deletion of 14 amino acids at the $N$-terminus. Wild type TPC6A is one of the components in the transport protein particle (TRAPP) complex in yeast [10-14]. In mouse, deletion of Trappc6a gene induces a phenotype with mosaic loss of coat pigment [13]. Subunits of TRAPP may exhibit independent functions in specific biological processes in mammals [14]. Human TRAPPC6A gene is involved in nonverbal reasoning in 2 Scottish cohorts, and is suggested for a role in $\mathrm{AD}$ [15].

We determined whether TPC6A $\Delta$ aggregation occurs in the hippocampi of postmortem middle-aged normal humans and AD patients. We showed that TPC6A physically interacted with tumor suppressor WW domain-containing oxidoreductase, designated as human WWOX or FOR, and mouse WOX1 [16-19; Reviews]. WWOX possesses two $N$-terminal WW domains, a $C$-terminal short chain alcohol dehydrogenase/reductase domain (SDR), and a nuclear localization signal in between the WW domains [16-19]. How WWOX blocked TPC6A $\Delta$ from self-aggregation, caspase activation and $\mathrm{A} \beta$ generation was examined. WWOX participates in embryonic neural development [20], and neuronal injury and damages [21-23]. WWOX inhibits GSK-3 $\beta$-mediated Tau hyperphosphorylation, and thus prevents AD pathogenesis [24]. Importantly, this event is needed for neuronal differentiation [24]. WWOX and isoform WOX2 are significantly downregulated in the AD hippocampus, and the downregulation leads to increased activities of enzymes in hyper-phosphorylating Tau [24-27].

\section{RESULTS}

\section{Isolation of an $\mathrm{N}$-terminal frame deletion isoform TPC6A $\Delta$ in mammalian cells}

TPC6A $\Delta$ was originally isolated from TGF- $\beta 1$ treated monocytic U937 cells in a subtraction cDNA library screen. This gene is expressed ubiquitously in many organs and tissues, according to the Genbank database. The deduced protein possesses an internal frame deletion of amino acids \#29-42 at the $N$-terminus
(Genbank accession FJ418644). The full-length or wild type TPC6A was constructed by inserting a missing DNA frame (42 bases) to the TPC6A $\triangle$ cDNA (Figure 1A). Selected amino acid sequence segments for generating specific polyclonal antibodies in rabbits against the fulllength TPC6A, TPC6A $\Delta$, and Ser35 phosphorylated TPC6A $\Delta$, respectively, are shown (Figure 1A).

To determine whether the generation of TPC6A $\triangle$ mRNA is caused by deletion of exon 1 of human TRAPPC6A gene, a primer set was designed to amplify a 213-base region comprising the target DNA segment (42 bp) and the flanking areas at both 5' and 3' ends (171 bp). The amplified DNA samples were subjected to sequence determination and shown to be identical to the sequence in the human TRAPPC6A gene. Thirty hippocampal samples, including 12 controls and $18 \mathrm{AD}$ patients from postmortem Caucasians, were examined. None of the genomic DNA samples were deleted in the exon 1 of human TRAPPC6A gene (Figure 1B). Similar results were observed by examining 50 genomic DNA samples in a random Asian population in Taiwan (data not shown).

Based upon the aforementioned observations, we determined whether TRAPPC6A mRNA undergoes alternative splicing. Computational analyses using 1,400 genomic sequences starting from the CDS sequence on TRAPPC6A exon 1 were performed [28, 29]. Results from 2 different web-based tools (NNSplice and NetGene) all predicted that the nt position 85 can be used as an alternative 5 ' donor site to initiate splicing and leads to a 42-bp deletion on TRAPPC6A exon 1 sequence. Additional evidence from EST database searching showed that 13 TPC6A cDNAs out of 55 in total in humans are with the 42-bp deletion. Proteins corresponding to wild type TPC6A and TPC6A $\Delta$ are shown in cells (Figure 2), which supports the occurrence of alternative splicing of TRAPPC6A mRNA. Data are provided to show the production of our homemade antibodies (Supplementary Figure 1). Also, the validity and protein aggregation are shown under various experimental conditions (Supplementary Figure 2 and 3).

\section{TPC6A $\Delta$ aggregates in human AD hippocampal and cortical tissues}

Unlike in the yeast [10-12], the functional properties of mammalian TPC6A are largely unknown. By immunohistochemistry (IHC), we showed the presence of extracellular TPC6A $\Delta$ aggregates or plaques with phosphorylation at Ser35 in the human AD cortex (Figure 2B-2D). In comparison, much less aggregation was shown for the wild type TPC6A in the brain cortex in age-matched control samples (Figure 2A). Specific antibody for the wild type TPC6A was used (Figure 2A). Pan specific antibody against both wild type and TPC6A $\triangle$ was also used to demonstrate aggregates in the cortex 


\section{B Brain genomic DNA}

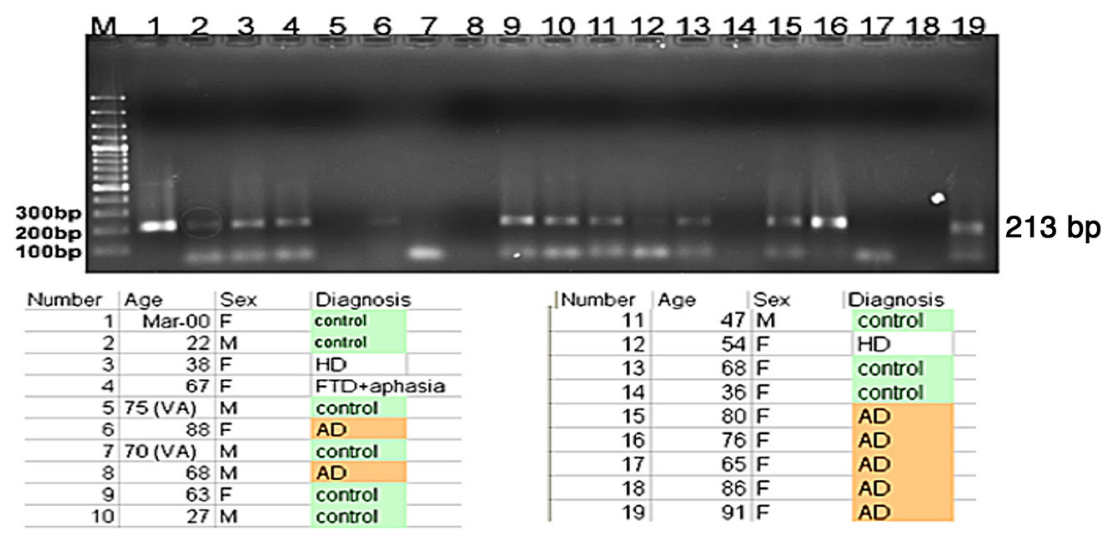

Figure 1: TRAPPC6A isoforms and gene. (A) Protein sequence alignment: 1) human wild type TRAPPC6A (TPC6A) (GenBank accession NP_077013), and 2) TPC6A $\Delta$ with a deletion of 14 amino acids at the $N$-terminus (GenBank accession FJ418644). Two potential phosphorylation sites are Ser49 and Tyr126 for TPC6A, or Ser35 and Tyr112 for TPC6A $\triangle$. Specific polyclonal antibodies were generated in rabbits against the underlined amino acid sequences: TPC6A (29-42) peptide (blue) antibody for wild type protein; TPC6A $\triangle$ (24-38) peptide (red) antibody for 17-kDa TPC6A $\Delta$ protein; pS35-TPC6A $\Delta$ (24-38) (red) peptide antibody for Ser35 phosphorylation of TPC6A $\Delta$; TPC6A (84-100) (green) peptide antibody for wild type and TPC6A $\triangle$. (B) Genomic DNA was isolated from indicated postmortem human hippocampal samples. A primer set was designed to amplify the possible deletion in the exon 1 and the flanking areas (total $213 \mathrm{bp}$, missing region 42 bp, \#17949610 to 17949569, Homo sapiens chromosome 19 genomic contig, GRCh37.p5 Primary Assembly), as shown in the mRNA encoding TPC6A $\triangle$. The amplified DNA samples were sequenced and found no deletions. A 90-bp DNA band at the bottom of the gel is a non-specific DNA fragment. "No signal" was due to failure of polymerase chain reaction (PCR).

of $\mathrm{AD}$ patients (Figure 2D). Our antibody is specific, as each immunizing peptide blocked the corresponding immunoreactivity (Figure 2A-2D). By Western blotting, the $20-\mathrm{kDa}$ wild type protein was identified by the specific antibody (duplicate loading; Figure 2A). Antibodies against TPC6A $\Delta$ and its phosphorylated form probed the $17-\mathrm{kDa}$ TPC6A $\Delta$ and polymerized pS35-TPC6A $\Delta$, respectively, in the neuroblastoma $\mathrm{SK}-\mathrm{N}-\mathrm{SH}$ cells (Figure 2B-2C). As determined using the pan-specific antibody, cytosolic wild type TPC6A is shown in the glial cells (Figure 2D). However, TPC6A became polymerized in the nucleus (Figure 2D).

\section{Aggregation of TPC6A and TIAF1 in nondemented human hippocampi}

By filter retardation assay, we have recently demonstrated the presence of water-insoluble TIAF1 aggregates in the hippocampi of nondemented humans at 40-75 years old, and the aggregates possess increasing amounts of $A \beta$ in the older AD samples (70-95 years old) [6]. Also, TIAF1 aggregation leads to the formation of $\mathrm{A} \beta$ in vitro [6]. Here, water-insoluble TPC6A aggregates were found in the hippocampi of postmortem humans, as determined by filter retardation assay (Figure 3A). Total samples from nondemented, younger controls (59 \pm 17 years old; $n=42)$ and older AD patients $(80 \pm 8.8$ years old; $n=96$ ) were randomly divided into 3 and 4 groups, respectively. TPC6A aggregates were found in both control and $\mathrm{AD}$ groups to a similar extent $(\sim 40 \%$ positive) (Figure 3A), suggesting that TPC6A aggregates are relatively stable in the brain. Similar results were observed with TIAF1 aggregates [6]. Both wild type TPC6A and TPC6A $\triangle$ are present in the aggregates, as determined using the TPC6A (84-100) peptide antibody. In agreement with our previous reports $[6,25]$, the levels of protein aggregates for Tyr33-phosphorylated WWOX (p-WWOX) were significantly reduced by $\sim 40 \%$ in the AD samples, compared to nondemented controls (Figure $3 \mathrm{~A})$. The extent of protein aggregation for NFT and $A \beta$ was significantly increased in the AD samples, compared with the nondemented controls (Figure 3A).

Next, we determined the presence of water-soluble aggregates using the aforementioned control and AD hippocampal samples by non-reducing SDS-PAGE and Western blotting. Large-sized TPC6A aggregates 


\section{Human brain cortex}

A

TPC6A
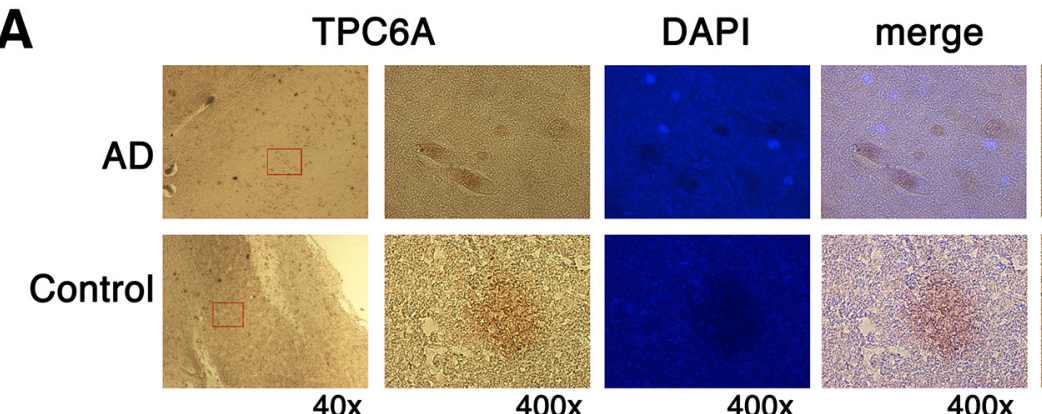

Peptide

blocking

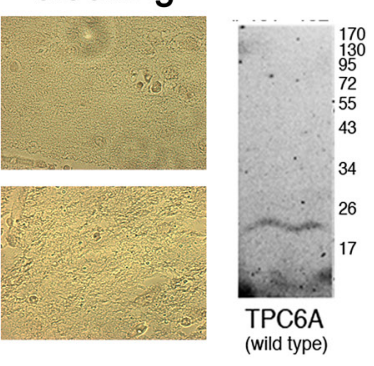

B
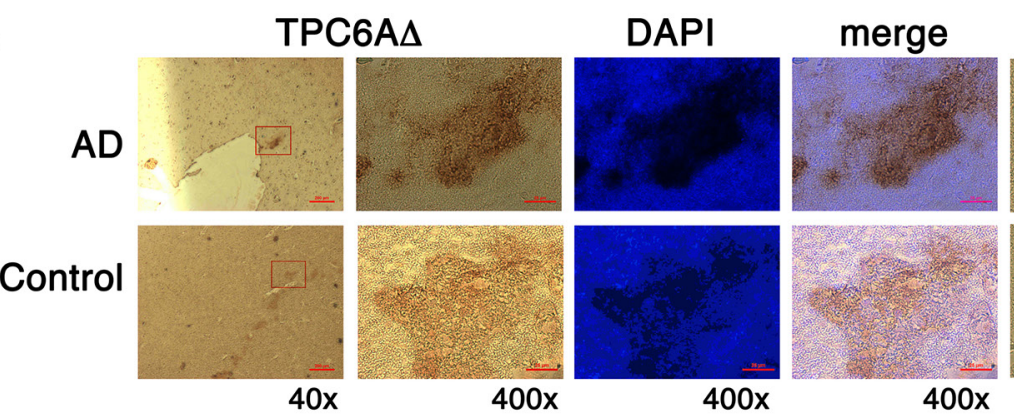

Peptide blocking
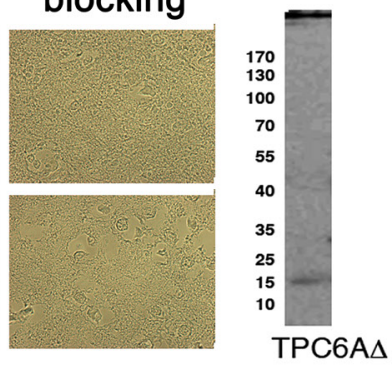

C
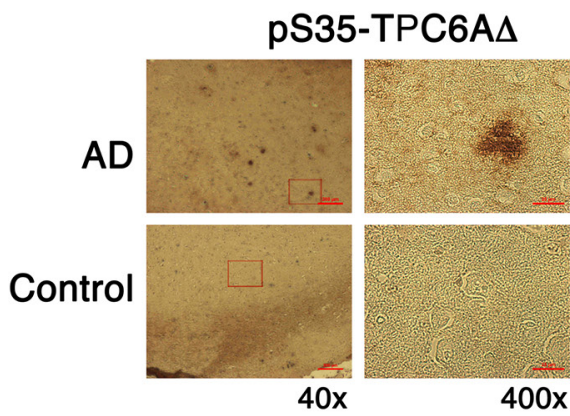

DAPI
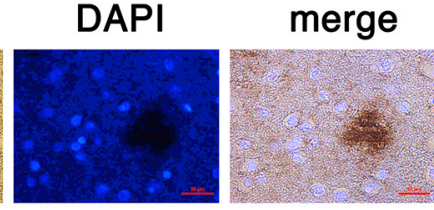

Peptide blocking
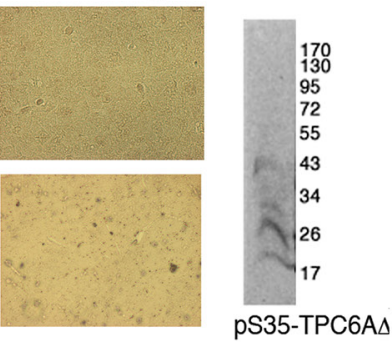

D
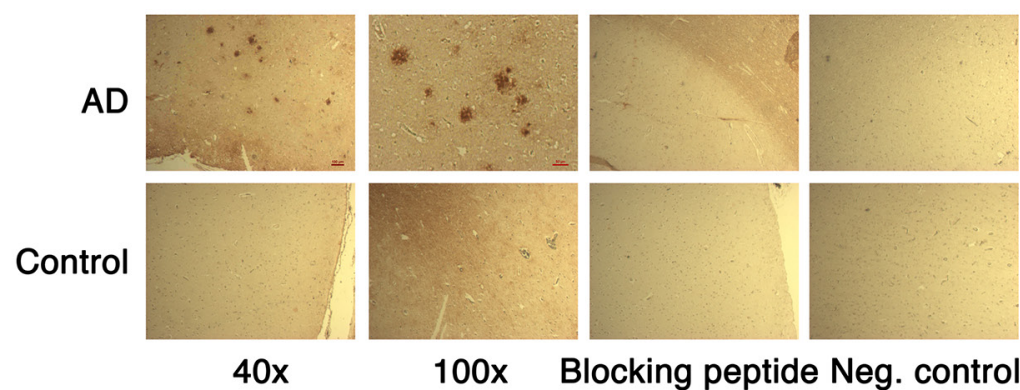

\section{$\frac{\text { Glial cells }}{\text { cytosol ncleus }}$}

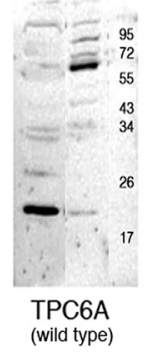

Figure 2: TPC6AD aggregates in the human brain cortex. Human brain cortical tissue sections from AD patients and agematched controls, along with lysates from cell lines, were used for IHC and Western blotting, respectively: (A) wild type 20-kDa TPC6A in human brain sections and wild type Wwox MEF cells stained with the wild type specific TPC6A (29-42) peptide antibody; (B) 17-kDa TPC6A $\Delta$ in human brain sections and SK-N-SH cells stained with the TPC6A $\Delta$ (24-38) peptide antibody; (C) pS35-TPC6A $\Delta$ in human brain sections and SK-N-SH cells stained with the pS35-TPC6A $\Delta$ (24-38) peptide antibody; (D) wild type and TPC6A $\Delta$ in human brain sections and mouse glial cells stained with the pan-specific TPC6A (84-100) peptide antibody. Nuclei were stained with DAPI. In controls, immunizing peptides were used to block the immunoreactivity. Also, in negative controls (see D), no primary antibodies were used in the IHC. Enlargements were made from boxed areas at 40x magnification to 400x. Scale bars for 40x, 100x, and 400x are 200, 100, and 25 $\mu \mathrm{m}$, respectively. 
A

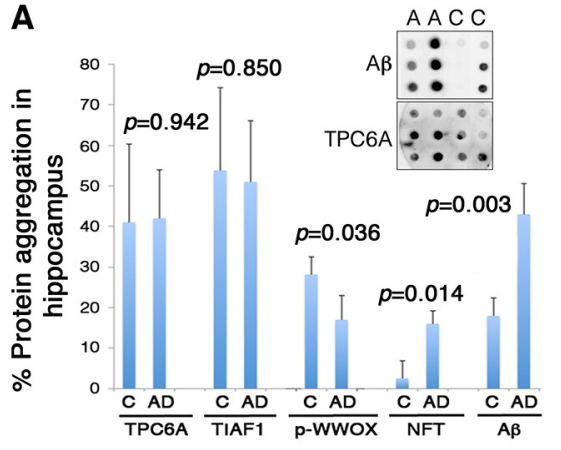

C

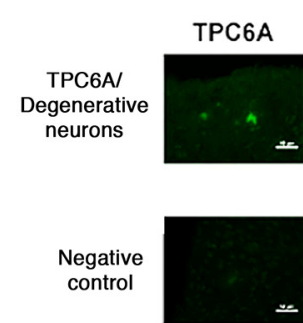

B

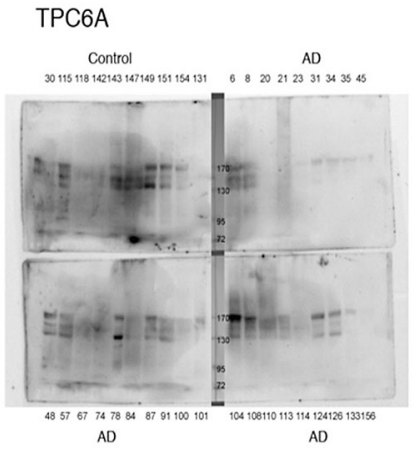

$\mathrm{AD}$

Fluoro-
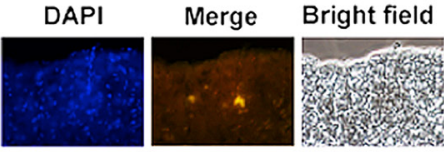

$200 X$
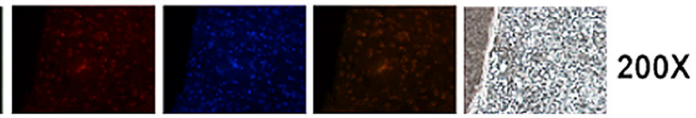

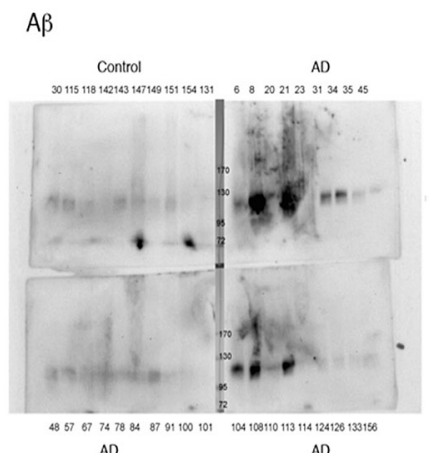

D

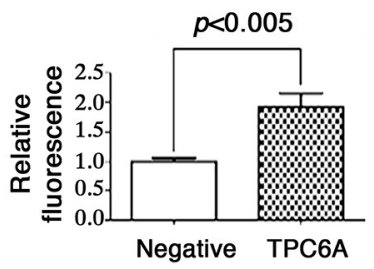

Figure 3: Soluble and insoluble TPC6A aggregates are present in the hippocampi of nondemented human controls and AD samples. (A) Frozen postmortem AD and control human hippocampal tissues were homogenized by sonication. After centrifugation, the insoluble pellets were further extracted and analyzed by filter retardation assay. Western blotting was then carried out using specific antibodies for TPC6A (pan-specific), TIAF1, Tyr33-phosphorylated WWOX (p-WWOX), NFT and A $\beta$. No significant difference was shown in TPC6A or TIAF1 aggregation for samples between younger nondemented controls (14 samples per group; total 3 groups; 42 to 76 years old) and older AD patients (24 samples per group; total 4 groups; 71 to 90 years old). C = control; A or AD = Alzheimer's disease. (B) The supernatants of the aforementioned samples were analyzed by 7\% non-reducing SDS-PAGE. Western blotting analyses show the presence of soluble aggregates of TPC6A and A $\beta$ in the representative protein preparations. Sample numbers are shown in each panel. Molecular weight markers: 170, 130, 95, $72 \mathrm{kDa}$. (C) Hippocampal tissue sections of APP/PS1-transgenic mice were stained with aliquots of TPC6A pan-specific antiserum and then with an Alexa Fluor 488-conjugated secondary antibody. Fluoro-Jade C was used for staining degenerative neurons, and nuclei stained with DAPI. TPC6A aggregates are localized in the degenerative neurons. A representative tissue section is shown. Scale bar: $50 \mu \mathrm{m}$. In negative controls, the sections were stained with the secondary antibody only, followed by staining with Fluoro-Jade C and DAPI. (D) Immuno-intensity was measured and normalized to negative controls (mean \pm standard deviation, $n=5 ; * *<0.05$, student's $t$ test).

are shown in both nondemented and $\mathrm{AD}$ hippocampi (Figure 3B). $\mathrm{A} \beta$ aggregates are more abundant in the $\mathrm{AD}$ hippocampal samples than in the controls (Figure 3B). We also showed the significantly increased levels of TPC6A aggregates in the degenerative neurons of hippocampi of APP/PS1-transgenic mice, as determined by immunofluorescence microscopy (Figure 3C-3D).

\section{TGF- $\beta 1$-induced TPC6A $\Delta$ aggregation for leading to caspase 3 activation and $A \beta$ production}

We determined whether aggregated TPC6A $\triangle$ causes caspase activation. Neuroblastoma SK-N-SH cells were transiently overexpressed with ECFP or ECFP-TPC6A $\triangle$. After culturing for $24 \mathrm{hr}$, the cells were treated with TGF- $\beta 1(5 \mathrm{ng} / \mathrm{ml})$ for $6-12 \mathrm{hr}$. TGF- $\beta 1$ significantly increased the production of $A \beta$ in the ECFP-TPC6A $\Delta$-expressing cells in $12 \mathrm{hr}$ (Figure 4A). We investigated the possible presence of aggregated TPC6A in the degenerative neurons. By staining AD hippocampal tissue sections, we showed the presence of TPC6A in the mitochondria of degenerated neurons (Figure 4A). Degenerative neurons were stained with Fluoro-Jade C [6], mitochondria with COX4 antibody, and TPC6A with the pan specific antibody. In the negative controls, no primary antibodies were used (Figure 4C). Presence of TPC6A in the mitochondria suggests that TPC6A may induce caspase activation during neuronal death.

Transiently overexpressed wild type TPC6A or TPC6A $\Delta$ was equally potent in causing cell death $(\sim 50$ $75 \%$ apoptosis of SK-N-SH and other cell lines using 10 $\mu \mathrm{g}$ expression constructs). When ectopic TPC6A $\Delta$ became aggregated in SK-N-SH cells, TPC6A $\triangle$ induced caspase 3 activation (Figure 4D). Without aggregation, TPC6A $\Delta$ did not induce activation of caspase 3 (Figure 4E). TGF- $\beta 1$ increased TPC6A $\triangle$ aggregation and subsequent caspase 3 activation (Figure $4 \mathrm{~F}$ ). In contrast, overexpressed wild type TPC6A, with or without aggregation, did not induce caspase 3 activation (Figure 4G-4H). 
A
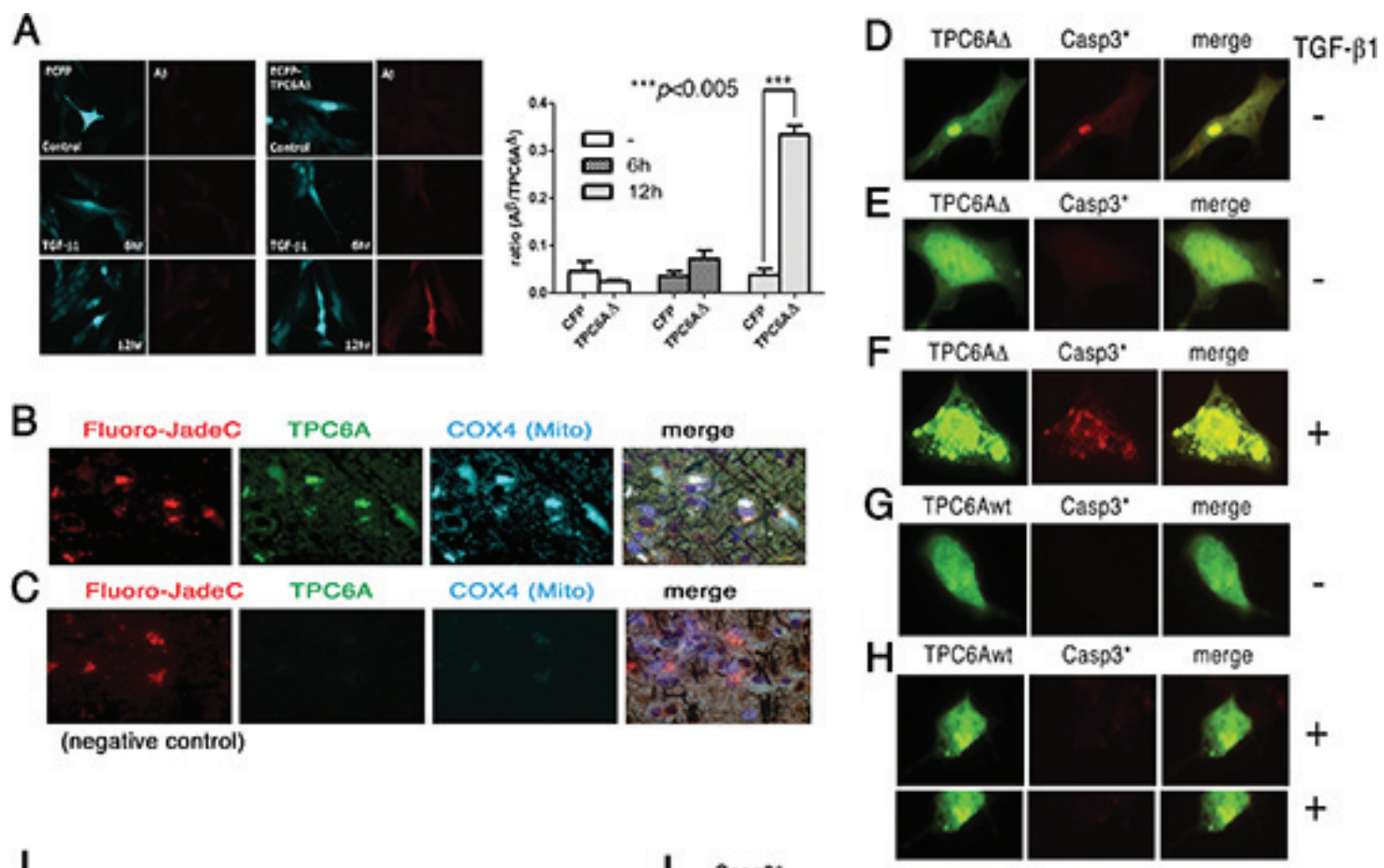

COX4 (Mito)

merge

(negative control)
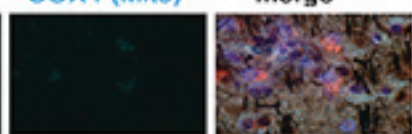

I
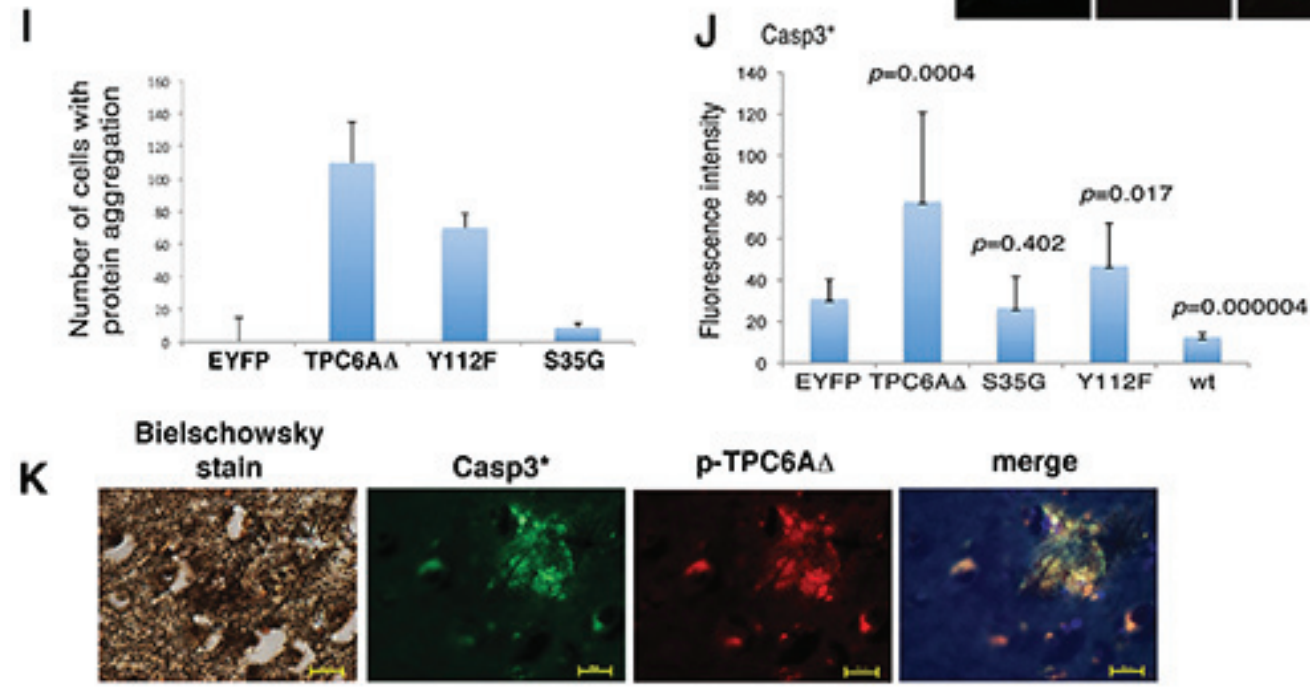

Figure 4: TGF- $\beta 1$ induces TPC6A $\Delta$ aggregation for leading to caspase activation. (A) Neuroblastoma SK-N-SH cells were transiently expressed with ECFP or ECFP-TPC6A $\Delta$ and treated with TGF- $\beta 1(5 \mathrm{ng} / \mathrm{ml})$ for indicated times, followed by fixation, permeabilization and staining with $\mathrm{A} \beta$ antibody. The ratios of the expression of A $\beta$ to TPC6A $\Delta$ are shown $(n=5)$. (B) TPC6A aggregates (green) are present in the mitochondria of degenerative neurons in the AD hippocampus. Tissue sections were pre-stained with the Bielschowsky stain (brown to dark), and then stained with Fluoro-Jade C Red for degenerative neurons (red), specific COX4 antibody for mitochondria (cyan), DAPI for nuclei (blue) and pan-specific antibody for TPC6A (green). 600x magnification; Scale bar: $10 \mu \mathrm{m}$. (C) In negative controls, no primary antibody was used in the immunostaining. (D) Transiently overexpressed EYFP-TPC6A $\Delta$ was aggregated in SK-N-SH cells, and caspase 3 became activated (Casp3*). (E) No caspase 3 activation was observed, if ectopic TPC6A $\Delta$ was not aggregated. (F) TGF- $\beta 1$ increased TPC6A $\triangle$ aggregation and caspase 3 activation. (G-H) Wild type TPC6A (TPC6Awt), either in a native or an aggregated state, did not induce caspase 3 activation. (I-J) SK-N-SH cells were transiently overexpressed with the indicated expression constructs. The number of cells harboring protein aggregation was counted, and the extent of caspase 3 activation was measured $(n=3$; Student's $t$ tests; $\sim 50$ cells counted per experiment). (K) Colocalization of S35-phosphorylated TPC6A $\Delta$ with activated caspase 3 in the AD hippocampal tissue sections. A representative data is shown.

By site-directed mutagenesis, the $\mathrm{S} 35 \mathrm{G}$ mutant of TPC6A $\Delta$ significantly lost its capability in aggregation and did not activate caspase 3 (Figure 4I-4J). The Y112F mutant had a reduced effect in aggregation and causing caspase 3 activation (Figure 4I-4J). Overexpressed wild type TPC6A underwent aggregation and caused apoptosis, but did not induce caspase 3 activation (Figure 4G-4J). In negative controls, EYFP did not undergo aggregation 
when overexpressed (Figure 4I-4J). S35-phosphorylated TPC6A $\triangle$ was shown to colocalize with activated caspase 3 in the human $\mathrm{AD}$ hippocampal tissue sections (Figure 4K).

\section{Wwox gene ablation induces TPC6А $\Delta$ and tau aggregation in the brain of 3-week-old knockout mice}

We have demonstrated that WWOX is significantly downregulated in the hippocampi of AD patients [24-26]. To evaluate the physiological significance, we generated Wwox gene knockout mice. Genotyping of the generated animals is shown (Figure 5A). The mice can only survive for about a month, which is in agreement with a previous report [30]. The wild type TPC6A is mainly expressed in the perinuclear areas of neurons in the brain cortex of 3-week-old knockout mice (Figure 5B). In contrast, TPC6A $\Delta$ aggregates are expressed in the extracellular matrix of the cortex (Figure 5B). Wild type TPC6A is expressed in the cytoplasm of neurons of the pyramidal layer of the hippocampus (see arrow; Figure 5C). However, pS35-TPC6A $\triangle$ and TPC6A $\Delta$ are located in the adjacent stratum oriens and stratum radiatum, and the proteins appear as extracellular aggregates (see arrows, Figure 5C; data not shown for TPC6A $\triangle$ ). Similarly, in cerebellum, wild type TPC6A is localized in the cytoplasm of Purkinje cells, whereas pS35-TPC6A $\Delta$ and TPC6A $\Delta$ are expressed in the matrix of white matter (data not shown). In parallel, pT181-Tau aggregates were significantly increased in the brain hemisphere sections of the 3-weekold Wwox knockout mice, as compared to wild type and heterozygous mice (Figure 5D).

\section{Knockdown of WWOX by siRNA induces aggregation of TPC6A $\Delta$ and TIAF1}

In parallel experiments, we examined the effect under WWOX knockdown. COS7 cells were cotransfected with small interfering RNA (siRNA)-targeting WWOX and EYFP-TPC6A $\triangle$, EYFP-TPC6A, or EYFP. The cells were then cultured for $24 \mathrm{hr}$. When WWOX was knocked down by siRNA (WOX1si), ectopic TPC6A $\Delta$ and TIAF 1 became aggregated by greater than $80 \%$ of cells $(\sim 100$ cells counted; Figure $6 \mathrm{~A}-6 \mathrm{D})$. In

A
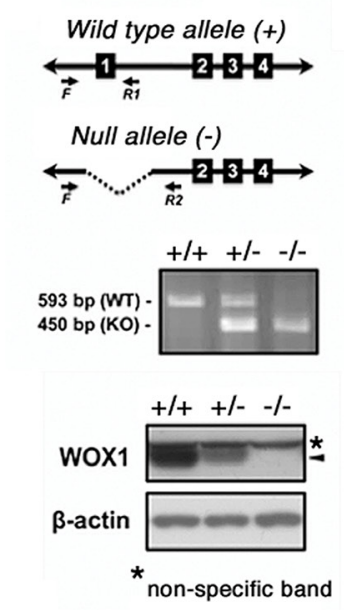

D PT181-Tau

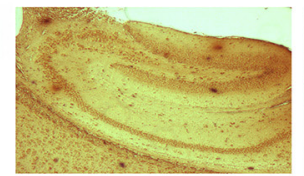

Wwox $-1-$
B
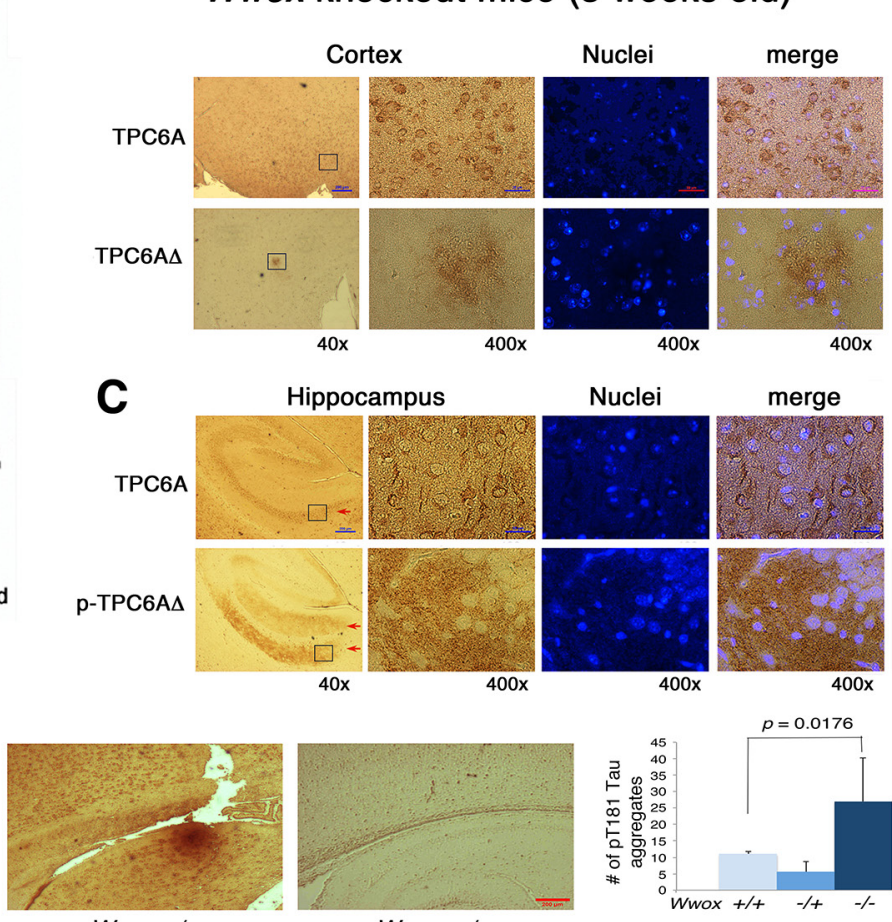

Wwox $-1-$
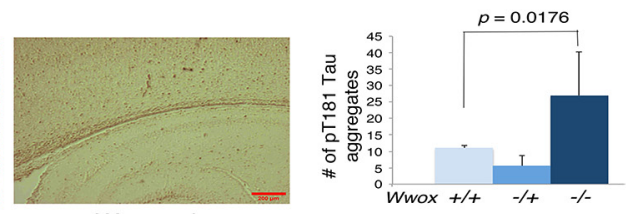

Figure 5: TPC6A $\Delta$ is an extracellular protein aggregate. (A) Ablation of $W$ wox gene at the exon 1 was carried out in mice. Genotyping of the generated animals and the expression of mouse WWOX/WOX1 protein (see arrow head) in MEF cells are shown. (B) The wild type TPC6A is mainly expressed in the perinuclear areas of neurons in the cortex of 3-week-old knockout mice. However, TPC6A $\Delta$ exhibits as extracellular aggregates in the brain cortex. (C) TPC6A is localized in the cytoplasm of neurons in the pyramidal layer of the hippocampus (see arrow).pS35-TPC6A $\Delta$ and TPC6A $\Delta$ are present in the extracellular matrix of adjacent stratum oriens and stratum radiatum as aggregates (see red arrows; data not shown for TPC6A $\Delta$ ). Enlargements were made from boxed areas at 40x magnifications to 400x. Scale bars for 40x and 400x are 200 and $25 \mu \mathrm{m}$, respectively. (D) The numbers of pT181-Tau aggregates were counted in the brain hemisphere sections of $W w o x+/+.-/+$, and $-/-$ mice $(n=5)$. Two representative pictures are shown for the brain tissue sections of Wwox $-/-$ mice, and one for $W$ wox $+/+$ mice. 

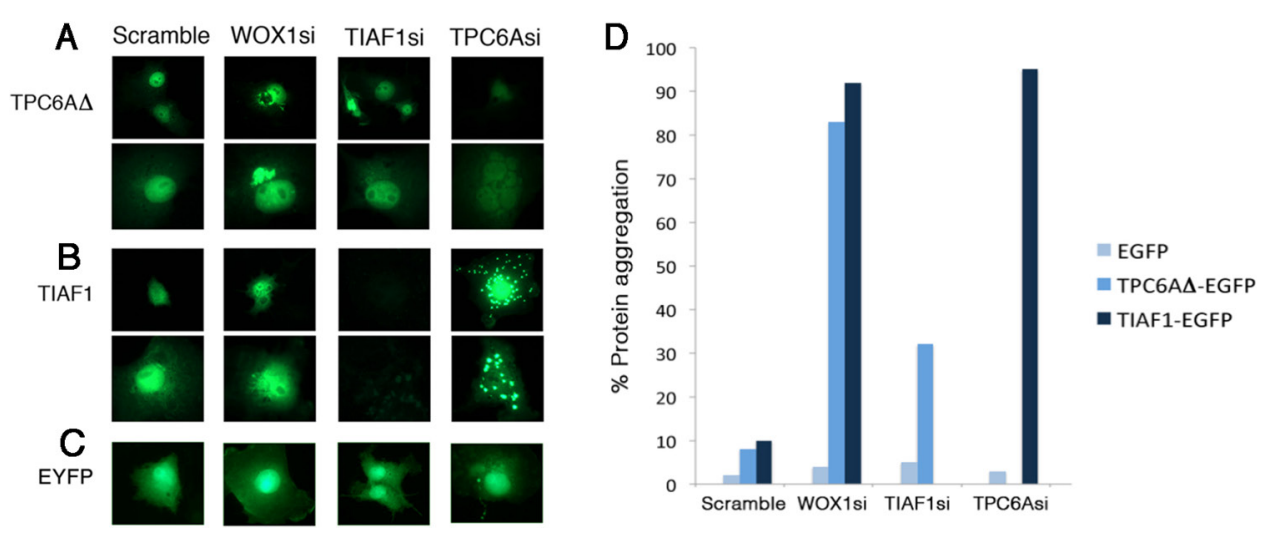

Figure 6: Induction of TPC6A and TIAF1 aggregation upon knockdown of endogenous WOX1 by siRNA. (A-C) COS7 cells were transfected with expression plasmid constructs for EYFP-TPC6A $\Delta$, EYFP-TIAF1, or EYFP, in the presence of one of the siRNA-expressing constructs, including scramble, WOX1si, TIAF1si and TPC6Asi. 24 hours later, protein aggregation was examined by fluorescent microscopy ( 150 cells counted). Data are shown in duplicates for (A) and (B). (D) Tabulated data is shown.

appropriate controls, no aggregation $(0 \%)$ was observed with EYFP alone in the presence of WOX1si or WWOXsi or scramble. Also, when cells were transfected with a "scramble DNA" construct, less than $10 \%$ protein aggregation was shown for TIAF1 and TPC6A (Figure 6A-6D). By time-lapse FRET microscopy, generation of cytosolic TPC6A $\triangle$ aggregates (see puncta) occurred when COS7 cells were transfected with WWOXsi (Video 1). However, no protein aggregation occurred when cells were transfected with a scrambled construct (Video 2). Together, the aforementioned observations are in parallel with the results from the mouse Wwox knockout model, suggesting that without WWOX in vitro and in vivo, TPC6A and TIAF1 start to polymerize or aggregate.

\section{DISCUSSION}

In summary, by utilizing specific antibodies, we have demonstrated for the first time that wild type TPC6A and isoform TPC6A $\triangle$ are expressed in distinct brain areas. TPC6A $\Delta$ is a plaque-forming protein in the brain extracellular matrix, whereas wild type TPC6A is a cytosolic protein. TPC6A, for instance, is expressed in the pyramidal layer and TPC6A $\triangle$ in the adjacent molecular layer of the hippocampus. TPC6A $\triangle$, but not the wild type, forms cortical plaques. TPC6A is abundant in the Purkinje cells of cerebellum, but TPC6A $\Delta$ is polymerized in the white matter. Most strikingly, TPC6A $\Delta$ and Tau plaques can be found in the brain cortex of $W$ wox knockout mice of less than 3 weeks old. WWOX is frequently downregulated in the hippocampi of AD patients [25, 26], suggesting that WWOX is crucial in preventing the aggregation of TPC6A $\Delta$ and Tau. We determined that TGF- $\beta 1$ causes dissociation of WWOX from TPC6A $\Delta$, thus leading to the aggregation of TPC6A $\triangle$ and TIAF1 and subsequent events including activation of caspases, and $A \beta$ production. In parallel, knockdown of WWOX causes aggregation of TIAF1 and TPC6A $\Delta$. Apparently, TPC6A $\Delta$ contributes a critical role in the aggregation of neuronal proteins and neurodegeneration.

In agreement with our previous observations [6], we determined that TPC6A $\Delta$ /TIAF1 aggregates or plaques are present in the hippocampi of normal individuals at midages, and the complexes possess increasing amounts of $\mathrm{A} \beta$ in the hippocampi of older AD patients. A critical finding from our study is that aggregating TPC6A $\triangle$ activates caspases and contributes, in part, to $A \beta$ generation. Thus, the ratio of wild type TPC6A versus TPC6A $\triangle$ isoform is likely to determine the tendency of AD pathogenesis in normal individuals. Caspase activation is known to contribute, in part, to the breakdown of APP and formation of A $\beta$ [31].

Whether TGF- $\beta 1$ regulates the binding of WWOX with TPC6A $\triangle$ is unknown and is being determined in this laboratory. Previously, we determined that TGF- $\beta 1$ induces the relocation of WWOX from the cytoplasm to the nucleus [32] and causes TIAF1 self-aggregation, A $\beta$ generation and apoptosis in certain cells [6]. Conceivably, WWOX is likely to complex with TPC6A $\Delta$, and TGF- $\beta 1$ would effectively increase their accumulation in the nucleus.

It is reasonable to assume that TPC6A aggregates serve as the nucleation sites for TIAF1 binding, caspase activation, and deposition of $A \beta$. Alteration of Ser35 to Gly35 in TPC6A $\triangle$ significantly abolishes the capability of TPC6A $\Delta$ in activating caspase 3 . TPC6A $\Delta$ plaques are mainly phosphorylated at Ser35 in the brain. These findings indicate that phosphorylation of TPC6A $\Delta$ is essential for causing neurodegeneration. In addition, TPC6A $\Delta$ /TIAF1 aggregation is shown in hippocampi of both postmortem $\mathrm{AD}$ patients and non-demented controls. The complexes appear to be stable in the brain, since their presence is found in the hippocampi of mid-aged normal individuals and older aged AD patients. TPC6A/ TIAF1 aggregates complex with $\mathrm{A} \beta$ deposits in the $\mathrm{AD}$ hippocampus, again suggesting that the aggregates are nucleation sites for $\mathrm{A} \beta$. 
WWOX is frequently downregulated in the hippocampus of AD patients [25]. We found that knockout Wwox-/- MEF cells are prone to possess aggregates of TPC6A, TIAF1, JNK1 and upregulated expression of $\beta$-secretase and Tau tangles (data not shown), suggesting a role of WWOX in stabilizing proteins and blocking their aggregation. Importantly, we demonstrated the presence of TPC6A plaques and pT181-Tau aggregates in the cortex of $W$ wox $-1-$ mouse brain. Wwox $-/-$ mice can only survive for one month. That is, plaques quickly form in less than a month. Recently, we demonstrated that WWOX physically interacts with GSK-3 $\beta$, and thereby suppresses Tau hyperphosphorylation [24]. WWOX also binds MEK1 for preventing the activation of ERK, thus reducing ERK-mediated phosphorylation of Tau [33]. Together, from previous and this studies, we have provided strong evidence for a crucial role of WWOX in preventing protein aggregation and neurodegeneration.

TPC6A is a subunit of TRAPP complex in yeast and mammals. In yeast, TPC6A is localized in ER and Golgi. In contrast, TPC6A is mainly localized in nuclei and the perinuclear regions in mammalian cells. The functional role TPC6A in the nuclei is unknown. A leucine zipper motif is predicted near the $C$-terminal of TPC6A, suggesting that TPC6A might be a DNA-binding protein. We predicted two possible phosphorylation sites in TPC6A $\Delta$, Ser35 and Tyr112. Alteration of Ser35 to Gly35 abolishes TGF- $\beta$-mediated aggregation of TPC6A $\Delta$. The Ser35 phosphorylation was verified by our produced antibody. Alteration of Tyr112 to Phe112 slightly reduces TPC6A $\Delta$ aggregation $(<30 \%)$ in the presence or absence of TGF- $\beta$. Importantly, overexpressed wild type TPC6A may undergo aggregation, but fails to activate caspases. Binding of TPC6A $\triangle$ with wild type TPC6A is weak, and may not cause generation of caspase activation (data not shown). However, both proteins become aggregated in the nucleolus upon stimulation with TGF- $\beta 1$.

WWOX prevents hyperphosphorylation of Tau by inhibiting GSK-3 $\beta$, ERK and other kinases, thereby preventing the formation of NFTs in neurons [24, 25]. Interestingly, blocking of GSK-3 $\beta$ activity by WWOX enhances neurite outgrowth and neuronal differentiation [24]. WWOX also binds Tau via its $C$-terminal SDR domain, whereas how the binding regulates the hyperphosphorylation of tau is unknown. The likely scenario is that WWOX may act as a chaperone, which stabilizes proteins from misfolding and being degraded by the ubiquitin/proteasome system.

\section{MATERIALS AND METHODS}

\section{Cell lines, chemicals and human postmortem hippocampal tissues}

Cell lines used in these studies were monkey kidney COS7 fibroblasts, human neuroblastoma SK-N-SH cells
(American Type Culture Collection), and isolated primary rat glial cells. Human postmortem frozen hippocampal tissues, as well as fixed tissue sections from hippocampi, were obtained from the Department of Pathology, University of Colorado Health Sciences Center (by Dr. CI Sze, before 2005) [6, 25]. IRB approval was waived. Informed consents were obtained from the family members of the deceased patients. Frozen hippocampal sections of APP/PS2 transgenic mice were prepared as described [6]. Fluoro-Jade C Red was from Chemicon/Invitrogen. The full length Zfra peptide was synthesized (GeneMed Synthesis).

\section{cDNA expression constructs, mutant clones, and transient expression}

TPC6A $\triangle$ was constructed in pECFP-C1, pEGFP-C1 and pEYFP-C1 (Clontech), respectively. The primers for these vectors were designed using a Mac DNASIS software (Hitachi), as follows: Forward primer, 5'-TCGAATTCTATGGCGGATACTGTGT; Reverse primer, 5'-ACGAATTCGATTAGGATTT CGGAATCAC. Site-directed mutagenesis was also employed to alter conserved phosphorylation sites Tyr112 and Ser35 using the following primer sets: 1) S35G, forward 5'-ATGAG CCTGGGAGTCCTGGA and reverse 5'-AGGTCCTGAGGGTCCGAGTA; 2) Y112F, forward 5'-TGGCCTGCAGTTTCTGGAGG and reverse 5'-ACCGGACGTCAAAGACCTCC. Expression constructs of full-length and truncated WWOX or WOX1 were made $[6,32]$. Transient overexpression of the indicated expression constructs in cells was performed by electroporation $\left(2 \times 10^{6}\right.$ cells; 200V, $50 \mathrm{~ms}$; BTX ECM 830 Square Wave Electroporator) or using GeneFector (Venn Nova), as described [6,34].

\section{Computational analysis}

To predict the possibility of alternative splicing on exon 1 of TRAPPC6A gene, online software developed with different algorithms was applied. The tools included Splice Site Prediction by Neural Network (NNSplice; http://www.fruitfly.org/seq_tools/splice.html) [35], and NetGene2 (http://www.cbs.dtu.dk/services/NetGene2/) [36].

\section{Isolation of TPC $6 A \Delta$ cDNA and construction of full-length TPC6A}

We constructed a cDNA library by treating human monocytic U937 cells with TGF- $\beta 1$. The TPC6A $\Delta$ cDNA was isolated from this library (with deletion at amino acids \#29-42; GenBank accession FJ418644). Full-length TPC6A-pEGFPC1 DNA was constructed by inserting synthetic primers into the TPC6A $\triangle \mathrm{cDNA}$. Primers, containing the deleted sequence of the 5 '-end 
truncation in TPC6A $\triangle$, were designed: forward, 5'-CCGACCCCGGCCCGGGGGTGAGCGCCGGGCT CCGTGG GGAGGAAGCGGGGGCCACCAAGG GACAGAAGATGAGCCTG; reverse, 5'-CAGGC TCATCTTCTGTCCCTTGGTGGCCCCCGCTTCCTC CCCACGGAGCCCGGCGCTCACCCCCGGGCCGG GGTCGG. At the 5' end, the primer has 18 bases corresponding to the TPC6Awt sequence, followed by the deleted 42 bases (bold) and then 17 bases for the 3' end. PCR was performed under the following cycling condition: step 1) heating at $94^{\circ} \mathrm{C}$ for $45 \mathrm{sec}$, step 2) cycling for 25 times at $94^{\circ} \mathrm{C}$ for $45 \mathrm{sec}, 65^{\circ} \mathrm{C}$ for $45 \mathrm{sec}$, and $72^{\circ} \mathrm{C}$ for $12 \mathrm{~min}$, and 3) final synthesizing at $72^{\circ} \mathrm{C}$ for $10 \mathrm{~min}$. The PCR product was digested with DpnI (New England BioLabs) at $37^{\circ} \mathrm{C}$ for $3 \mathrm{hr}$ to remove the original templates, and the amplified cDNA was transformed to $E$. coli Top 10 (Invitrogen). Positive clones were isolated and identified by sequencing analysis.

\section{Antibodies and antibody production in rabbits}

Use of rabbits for antibody production was approved by the Institutional Animal Care and Use Committee of the National Cheng Kung University Medical College. Four TPC6A or TPC6A $\Delta$ peptides were synthesized (Figure 1A): 1) CKDLWVAVFQKQMDSLR, amino acid \#84-100 for pan-specific antibody production; 2) CDPGPGGQKMSLSVLE, amino acid \#24-38 for antibody against TPC6A $\Delta$; 3) CDPGPGGQKMSLPSVLE, amino acid \#24-38 with phosphorylation at Ser35 for antibody against p-TPC6A $\Delta$; 4) CVSAGLRGEEAGATK, amino acid \#29-42 for antibody against wild type TPC6A. These peptides were conjugated with keyhole limpet hemocyanin (KLH) for antibody production in rabbits (Antibody Production and Purification kit from Pierce/ Invitrogen) $[6,20,25,27,34]$. The $N$-terminal cysteine in each peptide sequence was added to covalently conjugate with KLH. Specificity of the antisera was tested using the synthetic peptides to block immunostaining. Commercial antibodies used were against: A $\beta$ (AbD Serotec Cat\# MCA2172, RRID:AB_323833), APP (EMD Millipore) $[6,20,25]$, NFT (neurofibrillary tangles; Invitrogen) [25], $\alpha$-tubulin (Sigma-Aldrich), activated caspase 3 (Cell Signaling Technology), and TIAF1 (Abcam) [6, 37]. Antibodies against WWOX and Tyr33-phosphorylation in WWOX were made and purified as described [20, 25, 27, 32]. Adobe Photoshop CS5 software was used to analyze the extent of protein expression from Western blots.

\section{Immunohistochemistry, immunofluorescence, and time-lapse fluorescence microscopy}

De-parafinization, immunohistochemistry, and immunofluorescence staining were performed as described $[6,20-23,34,38,39]$.

\section{Filter retardation assay and protein aggregation} assay

Filter retardation assay was performed using postmortem human frozen hippocampal extracts [6]. Briefly, samples were homogenized in a protein lysis buffer, and centrifuged in a microfuge $\left(13,200 \mathrm{rpm}\right.$ for $30 \mathrm{~min}$ at $\left.4^{\circ} \mathrm{C}\right)$. The pellets were harvested and added $100 \mu \mathrm{l}$ DNase I buffer (20 mM Tris-HCl, pH 8.0, 15 mM MgCl, 1 mg/ml DNase I), and then incubated for $1 \mathrm{hr}$ at $37^{\circ} \mathrm{C}$. Protein preparations were quantified (BCA assay kit, Pierce). Each sample containing $10 \mu \mathrm{g}, 30 \mu \mathrm{g}$ and $60 \mu \mathrm{g}$ protein, respectively, was diluted in $100 \mu \mathrm{l}$ sample buffer (1\% SDS, 8\% $\beta$-mercaptoethanol in PBS), boiled for $10 \mathrm{~min}$ at $95^{\circ} \mathrm{C}$, and then filtered through $0.2 \mu \mathrm{m}$ cellulose acetate membranes using a dot-blot apparatus (BioRad). Each well was washed by $200 \mu \mathrm{l} 0.1 \%$ SDS twice. Then, the membranes were analyzed by standard Western blotting to determine the presence of A $\beta$, TPC6A and other proteins of interest.

\section{Development of Wwox knockout mice}

Generation of gene-targeting constructs and mouse chimera was performed at the Transgenic Mouse Models Core Facility, National Core Facility Program for Biotechnology at the National Taiwan University Medical College, Taipei (http://140.112.133.74/). Briefly, we have designed insertion of LoxP sites to a vector for targeting exon 1 and 2/3/4, respectively, by recombineering technology [40] (Supplementary Figure 4A-4B). Cremediated recombination of sequences flanked by LoxP sites was performed in embryonic stem (ES) cells [41]. The targeted ES cell clones were selected and injected into blastocysts to generate chimeric mice. Chimeras were crossed with $\mathrm{C} 57 \mathrm{BL} / 6$ mice to obtain germline transmission of the targeted allele. Heterozygous mice were then interbred to obtain wild-type $W w o x^{+/+}$, heterozygous $W w o x^{+/-}$, and homozygous knockout $W w^{\prime-1} x^{-1}$ mouse embryos. Mouse embryonic fibroblasts (MEF) were established from $\sim$ E16.5 mouse fetuses. PCR genotyping was performed using primer sequences (5'-tgagcttgggagaagtgggtactttg, 5 '-agctctatactatactggctggctgg, and 5'-aggtgttggaga cttctccactgcta for exon 1-deletion; 5'-gctctgtgaga ccatttggacagtgt, 5'-cttgattctgctgcctctgcttccta, and 5 '-cgagagaaggaagcctgttatctaga for exon 2/3/4-deletion) specific for the wild type or the targeted allele. All experimental procedures were carried out in accordance with an approved protocol for animal use from the Institutional Animal Care and Use Committee of National Cheng Kung University.

\section{PCR analysis of partial exon 1 deletion in human TRAPPC6A gene}

To determine possible frame deletion in TRAPPC6A gene, a primer set was designed to amplify 
a 213-base region flanking a genomic DNA segment (42 bases lacking in the TPC6A $\Delta$ cDNA) and a portion of non-deleted areas at both 5' and 3' ends: forward, 5'-GTTTCTTCACACGGAGATGG (in exon 1), and reverse, 5'-CCACTTTCCAAAGGAGGAAG (in intron $1-2)$. The optimal annealing temperature is $54-55^{\circ} \mathrm{C}$, as determined by gradient PCR (Mastercycler gradient, Eppendorf). The possibly deleted region in chromosome $19 q 13.32$ is 5'-GTGAGCGCCGGGCTCCGTGGGGA GGAAGCGGGGGCCACCAAG, encoding amino acid \#29-42 in the wild type TPC6A. Genomic DNA samples were purified from human normal and AD hippocampal tissues. The amplified DNAs were subjected to sequence determination.

\section{Data analysis}

Where indicated, all experiments were performed 3-5 times. Data were presented as mean \pm standard deviation. Student's $t$-tests were carried out for statistical analysis.

\section{ACKNOWLEDGEMENTS}

This research was supported, in part, by the Department of Defense USA (W81XWH-08-1-0682), the Ministry of Science and Technology, Taiwan, ROC (NSC98-2628-B-006-041-MY3， 99-2320-B-006-012MY3, 102-2320-B-006-018-, 102-2320-B-006-030-, and 102-3011-P-006-005-), the National Health Research Institute, Taiwan, ROC (NHRI-EX101-10102BI, and NHRI-EX102-10102BI), the National Cheng Kung University Landmark Projects (C0167) and the Department of Health, Taiwan, ROC (DOH101-TD-PB111-TM010) (to NS Chang). JYC was supported by the ASBMB Travel Award to the EB2009 conference.

\section{Conflict in interest statement}

The authors declare that there are no conflict in interest involved in conducting the research.

\section{REFERENCES}

1. Amijee H, Scopes DI. The Quest for Small Molecules as Amyloid Inhibiting Therapies for Alzheimer's Disease. J Alzheimers Dis. 2009; 17:33-47.

2. Iqbal K, Liu F, Gong CX, Grundke-Iqbal I. Tau in Alzheimer disease and related tauopathies. Curr Alzheimer Res. 2010; 7:656-664. Review.

3. Galimberti D, Scarpini E. Disease-modifying treatments for Alzheimer's disease. Ther Adv Neurol Disord. 2011; 4:203-216.

4. Salins P, He Y, Olson K, Glazner G, Kashour T, Amara F. TGF-betal is increased in a transgenic mouse model of familial Alzheimer's disease and causes neuronal apoptosis. Neurosci Lett. 2008; 430:81-86.

5. Town T, Laouar Y, Pittenger C, Mori T, Szekely CA, Tan J, Duman RS, Flavell RA. Blocking TGF-beta-Smad2/3 innate immune signaling mitigates Alzheimer-like pathology. Nat Med. 2008; 14:681-687.

6. Lee MH, Lin SR, Chang JY, Schultz L, Heath J, Hsu LJ, Kuo YM, Hong Q, Chiang MF, Gong CX, Sze CI, Chang NS. TGF- $\beta$ induces TIAF1 self-aggregation via type II receptor-independent signaling that leads to generation of amyloid $\beta$ plaques in Alzheimer's disease. Cell Death Dis. 2010; $1:$ e110.

7. Chang JY, Chiang MF, Lin SR, Lee MH, He H, Chou PY, Chen SJ, Chen YA, Yang LY, Lai FJ, Hsieh CC, Hsieh TH, Sheu HM, Sze CI, Chang NS. TIAF1 self-aggregation in peritumor capsule formation, spontaneous activation of SMAD-responsive promoter in p53-deficient environment, and cell death. Cell Death Dis. 2012; 3:e302.

8. Sze CI, Su WP, Chiang MF, Lu CY, Chen YA, Chang NS. Assessing current therapeutic approaches to decode potential resistance mechanisms in glioblastomas. Front Oncol. $2013 ; 3: 59$.

9. Hong Q, Hsu LJ, Chou PY, Chou YT, Lu CY, Chen YA, Chang NS. Self-aggregating TIAF1 in lung cancer progression. Translational Respiratory Medicine. 2013; 1:5.

10. Kümmel D, Müller JJ, Roske Y, Misselwitz R, Büssow K, Heinemann U. The structure of the TRAPP subunit TPC6 suggests a model for a TRAPP subcomplex. EMBO Rep. 2005; 6:787-793.

11. Kümmel D, Müller JJ, Roske Y, Henke N, Heinemann U. Structure of the Bet3-Tpc6B core of TRAPP: two Tpc6 paralogs form trimeric complexes with Bet3 and Mum2. J Mol Biol. 2006; 361:22-32.

12. Kümmel D, Oeckinghaus A, Wang C, Krappmann D, Heinemann U. Distinct isocomplexes of the TRAPP trafficking factor coexist inside human cells. FEBS Lett. 2008; 582:3729-3733.

13. Gwynn B, Smith RS, Rowe LB, Taylor BA, Peters LL. A mouse TRAPP-related protein is involved in pigmentation. Genomics. 2006; 88:196-203.

14. Sacher M, Kim YG, Lavie A, Oh BH, Segev N. The TRAPP complex: insights into its architecture and function. Traffic. 2008; 9:2032-2042. Review.

15. Hamilton G, Harris SE, Davies G, Liewald DC, Tenesa A, Starr JM, Porteous D, Deary IJ. Alzheimer's disease genes are associated with measures of cognitive ageing in the lothian birth cohorts of 1921 and 1936. Int J Alzheimers Dis. 2011; 2011:505984.

16. Chang NS, Hsu LJ, Lin YS, Lai FJ, Sheu HM. WW domaincontaining oxidoreductase: a candidate tumor suppressor. Trends Mol Med. 2007; 13:12-22.

17. Smith DI, McAvoy S, Zhu Y, Perez DS. Large common fragile site genes and cancer. Semin Cancer Biol. 2007; 17:31-41. Review. 
18. Del Mare S, Salah Z, Aqeilan RI. WWOX: its genomics, partners, and functions. J Cell Biochem. 2009; 108:737-745.

19. Chang JY, He RY, Lin HP, Hsu LJ, Lai FJ, Hong Q, Chen SJ, Chang NS. Signaling from membrane receptors to tumor suppressor WW domain-containing oxidoreductase. Exp Biol Med (Maywood). 2010; 235:796-804. Review.

20. Chen ST, Chuang JI, Wang JP, Tsai MS, Li H, Chang NS. Expression of WW domain-containing oxidoreductase WOX1 in the developing murine nervous system. Neuroscience. 2004; 124:831-839.

21. Chen ST, Chuang JI, Cheng CL, Hsu LJ, Chang NS. Lightinduced retinal damage involves tyrosine 33 phosphorylation, mitochondrial and nuclear translocation of WW domain-containing oxidoreductase in vivo. Neuroscience. 2005; 130:397-407.

22. Lo CP, Hsu LJ, Li MY, Hsu SY, Chuang JI, Tsai MS, Lin SR, Chang NS, Chen ST. MPP+-induced neuronal death in rats involves tyrosine 33 phosphorylation of WW domain-containing oxidoreductase WOX1. Eur J Neurosci. 2008; 27:1634-1646.

23. Li MY, Lai FJ, Hsu LJ, Lo CP, Cheng CL, Lin SR, Lee MH, Chang JY, Subhan D, Tsai MS, Sze CI, Pugazhenthi S, Chang NS, Chen ST. Dramatic co-activation of WWOX/ WOX1 with CREB and NF-kappaB in delayed loss of small dorsal root ganglion neurons upon sciatic nerve transection in rats. PLoS One. 2009; 4:e7820.

24. Wang HY, Juo LI, Lin YT, Hsiao M, Lin JT, Tsai CH, Tzeng YH, Chuang YC, Chang NS, Yang CN, Lu PJ. WW domain-containing oxidoreductase promotes neuronal differentiation via negative regulation of glycogen synthase kinase 3ß. Cell Death Differ. 2012; 19:1049-1059.

25. Sze CI, Su M, Pugazhenthi S, Jambal P, Hsu LJ, Heath J, Schultz L, Chang NS. Downregulation of WOX1 induces Tau phosphorylation in vitro: A potential role in Alzheimer's disease. J Biol Chem. 2004; 279:30498-30506.

26. Teng CC, Yang YT, Chen YC, Kuo YM, Sze CI. Role of WWOX/WOX1 in Alzheimer's disease pathology and in cell death signaling. Front Biosci (Elite Ed). 2012; 4:1951-1965.

27. Chang NS, Doherty J, Ensign A. c-Jun N-terminal kinase 1 (JNK1) physically interacts with WW domain-containing oxidoreductase (WOX1) and inhibits WOX1-mediated apoptosis. J Biol Chem. 2003; 278:9195-9202.

28. Buratti E, Chivers M, Královicová J, Romano M, Baralle M, Krainer AR, Vorechovsky I. Aberrant 5' splice sites in human disease genes: mutation pattern, nucleotide structure and comparison of computational tools that predict their utilization. Nucleic Acids Res. 2007; 35:4250-4263.

29. Kralovicova J, Christensen MB, Vorechovsky I. Biased exon/intron distribution of cryptic and de novo 3' splice sites. Nucleic Acids Res. 2005; 33:4882-4898.
30. Aqeilan RI, Trapasso F, Hussain S, Costinean S, Marshall D, Pekarsky Y, Hagan JP, Zanesi N, Kaou M, Stein GS, Lian JB, Croce CM. Targeted deletion of Wwox reveals a tumor suppressor function. Proc Natl Acad Sci U S A. 2007; 104:3949-3954.

31. Rohn TT, Head E. Caspase activation in Alzheimer's disease: early to rise and late to bed. Rev Neurosci. 2008; 19:383-393. Review.

32. Hsu LJ, Schultz L, Hong Q, Van Moer K, Heath J, Li MY, Lin SR, Lee MH, Lo CP, Lin YS, Chen ST, Chang NS. Transforming growth factor $\beta 1$ signaling via interaction with cell surface Hyal-2 and recruitment of WWOX/ WOX1. J Biol Chem. 2009; 280:16049-16059.

33. Lin HP, Chang JY, Lin SR, Lee MH, Huang SS, Hsu LJ, Chang NS. Identification of an in vivo MEK/WOX1 complex as a master switch for apoptosis in T cell leukemia. Genes Cancer. 2011; 2:550-562.

34. Chang NS, Pratt N, Heath J, Schultz L, Sleve D, Carey GB, Zevotek N. Hyaluronidase induction of a WW domaincontaining oxidoreductase that enhances tumor necrosis factor cytotoxicity. J Biol Chem. 2001; 276:3361-3370.

35. Reese MG, Eeckman FH, Kulp D, Haussler D. Improved splice site detection in Genie. J Comput Biol. 1997; 4:311-323.

36. Brunak S, Engelbrecht J, Knudsen S. Prediction of human mRNA donor and acceptor sites from the DNA sequence. J Mol Biol. 1991; 220:49-65.

37. Chang NS, Mattison J, Cao H, Pratt N, Zhao Y, Lee C. Cloning and characterization of a novel transforming growth factor- $\beta 1$-induced TIAF1 protein that inhibits tumor necrosis factor cytotoxicity. Biochem Biophys Res Commun. 1998; 253:743-749.

38. Chang NS, Doherty J, Ensign A, Schultz L, Hsu LJ, Hong Q. WOX1 is essential for tumor necrosis factor-, UV light-, staurosporine-, and p53-mediated cell death, and its tyrosine 33-phosphorylated form binds and stabilizes serine 46-phosphorylated p53. J Biol Chem. 2005; 280:43100-43108.

39. Lai FJ, Cheng CL, Chen ST, Wu CH, Hsu LJ, Lee JY, Chao SC, Sheen MC, Shen CL, Chang NS, Sheu HM. WOX1 is essential for UVB irradiation-induced apoptosis and down-regulated via translational blockade in UVBinduced cutaneous squamous cell carcinoma in vivo. Clin Cancer Res. 2005; 11:5769-5777.

40. Liu P, Jenkins NA, Copel NG. A highly efficient recombineering-based method for generating conditional knockout mutations. Genome Res. 2003; 13:476-484.

41. Tsai CW, Lai FJ, Sheu HM, Lin YS, Chang TH, Jan MS, Chen SM, Hsu PC, Huang TT, Huang TC, Sheen MC, Chen ST, Chang WC, Chang NS, Hsu LJ. WWOX suppresses autophagy for inducing apoptosis in methotrexatetreated human squamous cell carcinoma. Cell Death Dis. 2013; 4:e792. 